\title{
Sur le spectre des fibrés en tore qui s'effondrent
}

\author{
Pierre Jammes
}

RÉsumÉ.- On considère des fibrés en tores sur $S^{1}$ et $T^{2}$, munis d'une structure de solvariété, et on étudie le comportement du spectre du laplacien agissant sur les formes différentielles invariantes à gauche lors d'effondrements homogènes à courbure et diamètre bornés. On montre comment le nombre de petites valeurs propres dépend de la topologie du fibré et de la géométrie de l'effondrement. Mots clefs : effondrement, laplacien, formes différentielles, petites valeurs propres, nilvariétés, solvariétés.

Abstract. - We consider torus bundles over $S^{1}$ and $T^{2}$ with solmanifold structure, and analyze the behavior of the Laplacian acting on left-invariant differential forms under homogeneous collapsings with bounded diameter and bounded sectional curvature. We show how the number of small eigenvalues depends on the topology of the bundle and the geometry of the collapsing.

Keywords : collapsing, laplacian, differential forms, small eigenvalues, nilmanifolds, solvmanifolds.

MSC2000 : 58J50, 58C40

\section{Introduction}

Soit $(M, g)$ une variété riemannienne compacte connexe orientable de dimension $m$. On considère l'opérateur $\Delta=\mathrm{d} \delta+\delta \mathrm{d}$ agissant sur l'espace $\Omega^{p}(M)$ des $p$-formes différentielles sur $M$. Le spectre de cet opérateur forme un ensemble discret de nombres positifs ou nuls qu'on notera

$$
0=\lambda_{0, p}(M, g)<\lambda_{1, p}(M, g) \leq \lambda_{2, p}(M, g) \leq \ldots,
$$

où la multiplicité de $\lambda_{0, p}(M, g)$ est le $p$-ème nombre de Betti de $M$, les autres valeurs propres étant répétée s'il y a multiplicité.

L'étude du laplacien agissant sur les fonctions, i.e. le cas $p=0$, d'une variété riemannienne montre que si le diamètre et la courbure de Ricci de $M$ vérifient $\operatorname{diam}(M, g)<d$ et $\operatorname{Ric}(M, g) \geq-a g$, avec $a>0$, la première valeur propre non nulle est uniformément minorée ([Gr80],[LY80]) :

$$
\lambda_{1,0}(M, g)>C(m, a, d)>0 .
$$

Mais une telle inégalité ne se généralise pas aux $p>0$, même avec l'hypothèse plus forte de courbure sectionnelle bornée. Cependant, dans le cas où la courbure sectionnelle vérifie $|K(M, g)|<a$, et avec l'hypothèse supplémentaire que le rayon d'injectivité vérifie $\operatorname{inj}(M, g)>r$ avec $r>0$, B. Colbois et $\mathrm{G}$. Courtois ont montré ([CC90]) que

$$
\lambda_{1, p}(M, g)>C^{\prime}(m, a, d, r)>0 .
$$


Ce résultat a été amélioré par S. Chanillo et F. Trèves ([CT97]), qui obtiennent la minoration explicite

$$
\lambda_{1, p}(M, g)>C^{\prime \prime}(m, a, d) r^{4 m^{2}+4 m-2}, \text { avec } C^{\prime \prime}>0,
$$

où $r$ désigne le rayon d'injectivité de $M$. On voit que si une variété admet une suite de métrique à diamètre et courbure bornée telle que $\lambda_{1, p}$ tende vers 0 , pour $1 \leq p \leq m$, son rayon d'injectivité tend aussi vers 0 , c'est-à-dire qu'elle s'effondre.

Il n'est cependant pas clair en général, quand la variété s'effondre, de déterminer si $\lambda_{1, p}$ tend vers zéro. On sait toutefois, grâce aux travaux de R. Forman ([Fo95]), et de R. Mazzeo et R. Melrose ([MM90]) sur les limites adiabatiques d'un fibré - c'està-dire des effondrements consistant en des homothéties dans une direction verticale, la métrique restant constante dans la direction horizontale - , que l'existence de petites valeurs propres quand une variété s'effondre est fortement liée à sa topologie. Mais il nous semble aussi intéressant d'étudier des effondrements autres que les situations adiabatiques, et plus précisément d'observer comment varie le comportement du spectre en fonction de la géométrie de l'effondrement. Pour cela, nous allons considérer le cas simple de fibrés en tore $T^{n}$ munis d'une structure homogène - construits comme quotient $\Gamma \backslash G$ d'un groupe de Lie $G$ par un réseau cocompact $\Gamma$-, et étudier le comportement du spectre du laplacien $\Delta_{i n v}^{p}$ restreint à l'espace de dimension finie $\Omega^{p}(M)^{G}$ des $p$-formes homogènes - i.e. invariantes à gauche pour l'action de $G$ - lors d'effondrements par des métriques homogènes. En effet, les valeurs propres de $\Delta^{i n v}$, que nous noterons $\lambda_{k, p}^{i n v}(M, g)$ et qui sont en nombre fini, sont aussi valeurs propres de $\Delta$. En particulier, les petites valeurs propres de $\Delta^{i n v}$ sont petites valeurs propres de $\Delta$. De plus, J. Lott a montré réciproquement dans [Lo02] que dans le cas où $G$ est nilpotent, la recherche de petites valeurs propres de $\Delta$ se ramène à l'étude de $\Delta^{i n v}$ :

Proposition 1.1 (Lott). Il existe des constantes $a(n), a^{\prime}(n)$ et $c(n)$ strictement positives telles que si $M$ est une infranilvariété de dimension $n$ munie d'une métrique homogène pour laquelle $\|R\|_{\infty} \operatorname{diam}(M)^{2} \leq a^{\prime}$, où $\|R\|_{\infty}$ est la norme du tenseur de courbure, et si $\alpha$ est une forme propre du laplacien sur $M$ dont la valeur propre $\lambda$ vérifie $\lambda<a \operatorname{diam}(M)^{-2}-c\|R\|_{\infty}$, alors $\alpha$ est invariante.

Mais ce résultat ne se géneralise pas aux groupes résolubles. Nous en verrons un exemple au paragraphe 4.3 .

Dans un premier temps, nous allons étudier des exemples de fibrés en tore dont la base est un cercle et montrer que la topologie peut faire obstruction à l'existence de petites valeurs propres, et que de plus, dans le cas contraire, le nombre de valeurs propres tendant vers zéro dépend fortement de la géométrie de l'effondrement.

Exemple 1.2. Soit $G$ le groupe d'Heisenberg de dimension 3

$$
G=\left\{\left(\begin{array}{lll}
1 & x & z \\
0 & 1 & y \\
0 & 0 & 1
\end{array}\right), x, y, z \in \mathbb{R}\right\},
$$

et $\Gamma$ le sous-groupe de $G$ formé des matrices à coefficients entiers. Le quotient $M=$ $\Gamma \backslash G$ est une nilvariété. C'est aussi un fibré en tore $T^{2}$ sur le cercle dont les fibres sont les quotients des sous-variétés de $G$ d'équation $x=c^{t e}$. 
Soit $X, Y$ et $Z$ les champs de vecteurs invariants à gauche engendrés en $(0,0,0)$ par $\partial / \partial x, \partial / \partial y$ et $\partial / \partial z$. Ces champs passent au quotient sur $M$, le couple $(Y, Z)$ formant une base de l'espace vertical. Ils vérifient $[X, Y]=\mathrm{Z}$ et $[X, Z]=[Y, Z]=0$.

Soit $\varepsilon \in] 0,1]$ et $\alpha \geq 1$. On pose $X_{\varepsilon}=X, Y_{\varepsilon}=\varepsilon^{-1} Y$ et $Z_{\varepsilon}=\varepsilon^{-\alpha} Z$. On a alors $\left[X_{\varepsilon}, Y_{\varepsilon}\right]=\varepsilon^{\alpha-1} Z_{\varepsilon}$.

Soit $g_{\varepsilon}$ la métrique de $M$ invariante à gauche telle que la base $\left(X_{\varepsilon}, Y_{\varepsilon}, Z_{\varepsilon}\right)$ soit orthonormée en tout point. Quand $\varepsilon \rightarrow 0$, le diamètre de la fibre tend vers 0 . On déduit des crochets de Lie entre les vecteurs de la base que les formes de la base duale $\left(X_{\varepsilon}^{b}, Y_{\varepsilon}^{b}, Z_{\varepsilon}^{b}\right)$ vérifient

$$
\mathrm{d} X_{\varepsilon}^{b}=0, \mathrm{~d} Y_{\varepsilon}^{b}=0 \text { et } \mathrm{d} Z_{\varepsilon}^{b}=\varepsilon^{\alpha-1} X_{\varepsilon}^{b} \wedge Y_{\varepsilon}^{b}
$$

et le calcul montre finalement que

$$
\Delta X_{\varepsilon}^{b}=0, \Delta Y_{\varepsilon}^{b}=0 \text { et } \Delta Z_{\varepsilon}^{b}=\varepsilon^{2(\alpha-1)} Z_{\varepsilon}^{b} .
$$

On voit que si $\alpha=1$ (situation adiabatique), le spectre de $\Delta_{i n v}^{1}$ est indépendant de $\varepsilon$ mais si $\alpha>1$, alors $\lambda_{1,1}^{i n v}$ tend vers zéro quand $\varepsilon$ tend vers zéro.

Le fait qu'une variété qui s'effondre sur un cercle admette une structure de solvariété est déjà connu ([Pe89], [Tu97]). Nous nous proposons ici d'en donner une construction explicite dans un cas simple. Les fibrés considérés seront définis comme suspension d'un difféomorphisme linéaire de la fibre $T^{n}$ représenté par un élément $A \in \mathrm{SL}_{n}(\mathbb{Z})$ (comme $A$ est à coefficients entiers, l'action de $A$ sur $\mathbb{R}^{n}$ laisse stable le réseau $\mathbb{Z}^{n}$, et donc passe au quotient sur $T^{n}$ ). Par exemple, en 1.2 , cette matrice est $\left(\begin{array}{ll}1 & 1 \\ 0 & 1\end{array}\right)$. De plus, nous ferons l'hypothèse simplificatrice que $A$ admet un logarithme réel - en particulier pour représenter facilement le groupe $G$ dans $\mathrm{GL}_{n+2}(\mathbb{R})$ - , ce qui laissera malgré tout un grand nombre d'exemples à notre disposition. Enfin, au lieu de prendre une borne sur le diamètre comme hypothèse de normalisation, nous fixerons la métrique de la base.

Les propriétés du spectre que nous allons mettre en évidence sont données par le

Théorème 1.3. Soit $A \in \mathrm{SL}_{n}(\mathbb{Z})$ et $B \in \mathrm{GL}_{n}(\mathbb{R})$ tels que $A=\exp (B)$, d la dimension du sous-espace caractéristique associé à la valeur propre 0 de $B$ et d' la dimension de son noyau. Il existe un groupe $G(B) \subset \mathrm{GL}_{n+2}(\mathbb{R})$ et un réseau $\Gamma \subset G$ tel que $\Gamma \backslash G$ soit homéomorphe au fibré $M$ de fibre $T^{n}$ de fibration $p: M \rightarrow S^{1}$ construit par suspension du difféomorphisme A. Si de plus on suppose que les métriques sur $M$ sont homogènes et telles que p soit une submersion riemannienne pour une métrique de volume 1 sur $S^{1}$, alors :

1.3.1 $\operatorname{dim} \operatorname{Ker} \Delta_{i n v}^{1}=d^{\prime}+1$ et $\Delta_{\text {inv }}^{1}$ admet $n-d^{\prime}$ valeurs propres non nulles distinctes ou non.

1.3.2 Pour tout $a>0$, il existe une constante $c(B, a)>0$ telle que pour toute métrique invariante $g$ sur $M$ telle que la courbure sectionnelle vérifie $|K(M, g)|<a$, on a $\lambda_{i, 1}^{i n v}(M, g)<c$, pour tout $i$.

1.3.3 Si $d \neq n$, alors pour tout $a>0$, il existe une constante $c^{\prime}(B, a)>0$ telle que pour toute métrique $g$ sur $M$ la courbure sectionnelle vérifie $|K(M, g)|<a$, on $a \lambda_{d-d^{\prime}+1,1}^{i n v}(M, g)>c^{\prime}$. 
Si $d=n$ et $d^{\prime} \neq n$, alors $G$ est nilpotent, et il existe une suite de métriques $g_{\varepsilon}$ sur $M$ telle que la courbure soit uniformément bornée et $\lambda_{i, 1}^{i n v}\left(M, g_{\varepsilon}\right) \rightarrow 0$ quand $\varepsilon \rightarrow 0$, pour tout $0<i \leq n-d^{\prime}$.

Si $d=d^{\prime}=n$, alors $G=\mathbb{R}^{n}$, $M$ est un tore et les formes harmoniques sont exactement les formes invariantes.

1.3.4 Pour tout $k \leq d-d^{\prime}$, il existe une famille de métriques $g_{\varepsilon}^{k}$ sur $M$ de courbure et diamètre uniformément bornés par rapport à $\varepsilon$ et une constante $c^{\prime \prime}(B, k)>0$ telle que $\lambda_{i, 1}^{i n v}\left(M, g_{\varepsilon}^{k}\right) \rightarrow 0$ pour $i \leq k$ quand $\varepsilon \rightarrow 0$, et $\lambda_{k+1,1}^{i n v}\left(M, g_{\varepsilon}^{k}\right)>c^{\prime \prime}$ si $k<n$.

Remarque 1.4. Le point 1.3.4 montre que le nombre de petites valeurs propres peut, quand la topologie le permet, fortement varier avec la géométrie de l'effondrement.

Remarque 1.5. La démonstation de 1.3 .4 met en évidence le fait que dans le cas d'un effondrement par homothéties de la fibre, il n'y a pas de petites valeurs propres.

D'autre part, ce théorème permet de donner une condition nécessaire et suffisante sur $B$ pour l'existence de petites valeurs propres pour les 1-formes :

Corollaire 1.6. Sous les hypothèses du théorème 1.3, il existe une suite de métriques homogènes $g_{\varepsilon}$ sur $M$ telle que $\lambda_{1,1}^{\text {inv }}\left(M, g_{\varepsilon}\right) \rightarrow 0$ quand $\varepsilon \rightarrow 0$ si et seulement si $d \neq d^{\prime}$ (i.e. si la réduite de Jordan de $B$ a un bloc nilpotent non nul).

Remarque 1.7. Ce résultat est illustré par l'exemple 1.2 , pour lequel on a $B=$ $\left(\begin{array}{ll}0 & 1 \\ 0 & 0\end{array}\right), d=2$ et $d^{\prime}=1$.

Remarque 1.8. L'existence d'un bloc nilpotent non nul dans la réduite de Jordan de $B$ implique l'existence d'un bloc unipotent non trivial dans la réduite de $A$, ce qui nous donne une condition nécessaire sur la topologie. Notons aussi que cette condition sur $A$ est un cas particulier d'un résultat récent de J. Lott ([Lo02], corollaire 4).

Remarque 1.9. Les résultats 1.3 et 1.6 montrent que le comportement asymptotique du spectre de $\Delta_{i n v}^{1}$ est essentiellement lié à la nature, s'il existe, du bloc nilpotent de la réduite de Jordan de $B$.

Dans le cas des $p$-formes, $p \geq 2$, la situation est plus complexe, et en particulier le corollaire 1.6 n'est pas vrai pour $n$ et $p$ quelconque (on verra en 4.1 un exemple montrant qu'il peut exister une petite valeur propre pour les 2 -formes alors que $B$ n'a pas de bloc nilpotent). On peut cependant donner une condition nécessaire à l'existence de petites valeurs propres, à savoir que $B$ n'est pas semi-simple. En effet, on a le :

Théorème 1.10. Sous les hypothèse du théorème 1.3, si B est semi-simple, alors il existe $c^{\prime \prime}(B, a)>0$ tel que pour toute métrique homogène $g$ sur $M$ dont la courbure sectionnelle vérifie $|K(M, g)|<a$, on a $\lambda_{1, p}^{i n v}(M, g)>c^{\prime \prime}$. 
Dans [Lo02], J. Lott montre un résultat semblable pour un fibré en tore sur une base quelconque (théorème 6), mais avec des hypothèses plus fortes sur la structure du fibré.

En petite dimension, on peut être plus précis et mettre en évidence un lien simple entre l'existence de petites valeurs propres et la structure du groupe $G$ :

Corollaire 1.11. Supposons que $n=2$ ou 3 . S'il existe $p \in[1, n]$ et une suite de métriques homogènes sur $M$ telle que la courbure sectionnelle associée soit uniformément bornée et que $\lambda_{1, p}^{i n v}$ tende vers 0 , alors $G$ est nilpotent.

Remarque 1.12. C'est par exemple la situation exposée en 1.2 , où on a $p=1$ et $n=2$.

Les résultats 1.3, 1.6, 1.10 et 1.11 seront démontrés dans la partie 2 .

Dans un deuxième temps, nous étudierons le cas des fibrés principaux en tore $T^{n}$ dont la base est un tore $T^{2}$. Leur topologie est relativement simple. On peut par exemple les construire comme somme de Whitney ([Hu66], p.15) de $n$ fibrés en cercle sur $T^{2}$. Nous allons montrer qu'un tel fibré est aussi difféomorphe au produit d'un tore et d'une nilvariété de dimension 3, pour mettre ensuite en évidence le fait que s'il est muni d'une métrique homogène, il existe une unique valeur propre non nulle sur les formes invariantes qu'on peut calculer en fonction de la métrique.

On obtient le résultat suivant :

Théorème 1.13. Soit $M$ un fibré principal non trivial en tore $T^{n}$ sur le tore $T^{2}$. Alors

1.13.1 $M$ est une nilvariété et, si $n \geq 2, M$ est homéomorphe à $N \times T^{n-1}$, où $N$ est une nilvariété de dimension 3.

1.13.2 Il existe un vecteur $V$ vertical tel que si $M$ est muni d'une métrique homogène, alors pour tout $p \in[1, n+1], \Delta_{\text {inv }}^{p}$ admet une unique valeur propre non nulle $\lambda$. Sa multiplicité est $C_{n}^{p-1}$, et $\lambda=\operatorname{Vol}(B)^{-2}|V|^{2}$, où $\operatorname{Vol}(B)$ est le volume de la base du fibré pour la métrique induite.

Remarque 1.14. Le produit du 1.13.1 n'est pas nécessairement riemannien pour les métriques considérées. Le spectre ne peut donc pas se déduire de la formule de Künneth.

Remarque 1.15. On voit que contrairement à la situation du théorème 1.3 , un effondrement par homothéties de la fibre produit une petite valeur propre, et que $\lambda$ est alors proportionnel au carré du diamètre de la fibre, à topologie fixée.

Remarque 1.16. Dans le cas où $n=1$, la remarque précédente rejoint les résultats de B. Colbois et G. Courtois qui étudient dans [CC00] le spectre des fibrés en cercles sur des bases quelconques et sans restrictions sur la métrique. Mais si la dimension de la fibre est plus grande $(n \geq 2)$, un phénomène nouveau apparaît : il existe dans ce cas des effondrements du fibré tels que $\lambda$ ne tende pas vers zéro. Nous en donnerons des exemples au paragraphe 4.2. Si $n \geq 2$, le nombre de petites valeurs propres ne dépend donc pas uniquement de la topologie. Cependant, on a pas de liberté sur ce nombre comme en 1.3.4.

Le théorème 1.13 sera démontré dans la partie 3 . 


\section{Fibrés en tore sur le cercle}

\subsection{Structure homogène}

Nous commençons par démontrer le début du théorème 1.3 en construisant le groupe $G$ et le réseau $\Gamma$ qui nous intéressent. Considérons un fibré $M$ en tore $T^{n}$ sur le cercle qui est la suspension d'un difféomorphisme linéaire $\varphi$ représenté par la matrice $A \in \mathrm{SL}_{n}(\mathbb{Z})$. Un tel fibré sera homéomorphe à

$$
M:=T^{n} \times[0,1] /(x, 0) \sim(\varphi(x), 1),
$$

Pour construire $G$, on va munir $\mathbb{R}^{n+1}$ d'une structure de groupe telle que $\mathbb{Z}^{n+1} \mathbb{R}^{n+1}=$ $M$. Si on note $\left(x_{1}, \cdots, x_{n}, y\right)$ les éléments de $\mathbb{R}^{n+1}$, une telle structure devra vérifier

$$
\left(k_{1}, \cdots, k_{n}, 0\right) \cdot\left(x_{1}, \cdots, x_{n}, y\right)=\left(x_{1}+k_{1}, \cdots, x_{n}+k_{n}, y\right)
$$

de sorte que les sous-espaces de $\mathbb{R}^{n+1}$ d'équation $y=c^{t e}$ passent au quotient comme des tores $T^{n}$, et

$$
(0, \cdots, 0, l) \cdot\left(x_{1}, \cdots, x_{n}, y\right)=\left(A^{l}\left(\begin{array}{c}
x_{1} \\
\vdots \\
x_{n}
\end{array}\right), y+l\right)
$$

de sorte que la structure de fibré soit bien celle définie par (2.1). Cette structure est effectivement réalisée en définissant $G$ comme l'image du le plongement

$$
\left(x_{1}, \cdots, x_{n}, y\right) \longmapsto\left(\begin{array}{c|cc}
A^{y} & \begin{array}{c}
0 \\
\vdots
\end{array} & x_{1} \\
& \vdots & \vdots \\
0 & 1 & y \\
\hline 0 & 0 & 1
\end{array}\right) .
$$

Comme on se restreint aux matrices $A$ qui admettent un logarithme $B$, l'expression $A^{y}$ est bien définie en posant $A^{y}=\exp (y B)$. On peut facilement vérifier que cette application est injective, que son image $G$ est bien un sous-groupe de $G_{n+2}(\mathbb{R})$ et que sa structure est bien celle définie par (2.2) et (2.3). Enfin, l'image de $\mathbb{Z}^{n+1}$ par cette application est bien un sous-groupe discret de $G$, qu'on notera $\Gamma$. La variété $M$ est donc homéomorphe au quotient $\Gamma \backslash G$.

Remarque : on peut vérifier que si $A=\left(\begin{array}{ll}1 & 1 \\ 0 & 1\end{array}\right)$, le groupe $G$ obtenu est isomorphe au groupe d'Heisengerg de dimension 3 tel qu'il est présenté dans l'exemple 1.2.

\subsection{Laplacien}

Soit $X_{i}$ et $Y$ les champs invariants à gauche engendrés en $I_{n+2}$ respectivement par

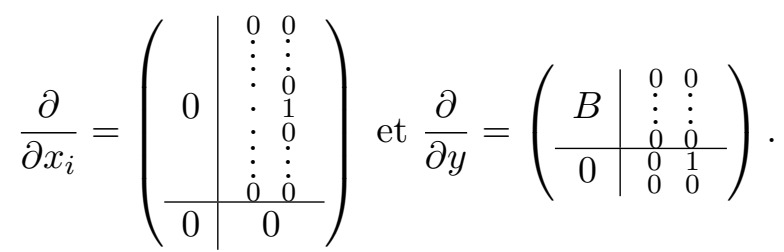

Ces champs vérifient $\left[X_{i}, X_{j}\right]=0$ et $\left[Y, X_{i}\right]=\sum_{j=1}^{n} b_{j i} X_{j}$. On peut remarquer que l'application $X \mapsto[Y, X]$ est un endomorphisme de l'espace $\Gamma\left(T_{V} M\right)^{G}$ des formes 
invariantes verticales, c'est-à-dire l'espace engendré par les $X_{i}$, et dont la matrice est $B$. On notera $f$ cet endomorphisme.

On fixe une métrique homogène $g$ sur $M$ en se donnant une base $\left(V_{i}\right)$ de l'espace $\Gamma\left(T_{V} M\right)^{G}$, cette métrique étant telle que $\left(V_{1}, \cdots, V_{n}, Y\right)$ soit orthonormée en tout point. On notera $\left(V_{1}^{b}, \cdots, V_{n}^{b}, Y^{b}\right)$ sa base duale, et $C$ la matrice de $f$ dans la base $\left(V_{1}, \cdots, V_{n}\right)$. On va déterminer le spectre du laplacien $\Delta_{i n v}^{1}$ restreint à l'ensemble $\Omega^{1}(M)^{G}$ des 1-formes invariantes à gauche en fonction des coefficients de $C$. Plus précisément, on a le

Lemme 2.4. La matrice du laplacien $\Delta_{i n v}^{1}$ dans la base $\left(V_{1}^{b}, \cdots, V_{n}^{b}, Y^{b}\right)$ est

$$
\Delta_{\text {inv }}^{1}:\left(\begin{array}{cc}
C^{t} C & 0 \\
\vdots \\
0 \cdots 0 & 0
\end{array}\right) .
$$

Démonstration : Les crochets de Lie entre les vecteurs de la nouvelle base sont

$$
\left[V_{i}, V_{j}\right]=0 \text { et }\left[Y, V_{i}\right]=\sum_{j=1}^{n} c_{j i} V_{j} .
$$

Soit $\alpha$ une 1-forme différentielle. Sa différentielle extérieure est donnée par la relation $\mathrm{d} \alpha\left(U_{1}, U_{2}\right)=U_{1} \cdot \alpha\left(U_{2}\right)-U_{2} \cdot \alpha\left(U_{1}\right)-\alpha\left(\left[U_{1}, U_{2}\right]\right)$, où $U_{1}$ et $U_{2}$ sont des champs de vecteur. Si ces champs sont invariants à gauche, cette relation devient : $\mathrm{d} \alpha\left(U_{1}, U_{2}\right)=$ $-\alpha\left(\left[U_{1}, U_{2}\right]\right)$. On en déduit :

$$
\mathrm{d} Y^{\mathrm{b}}=0 \text { et } \mathrm{d} V_{i}^{\mathrm{b}}=-\sum_{j=1}^{n} c_{i j} Y^{\mathrm{b}} \wedge V_{j}^{\mathrm{b}} .
$$

La matrice de la différentielle extérieure d : $\Omega^{1}(M)^{G} \rightarrow \Omega^{2}(M)^{G}$ sera, dans les bases $\left(V_{1}^{b}, \cdots, V_{n}^{b}, Y^{b}\right)$ et $\left(Y^{b} \wedge V_{i}^{b}, V_{i}^{b} \wedge V_{j}^{b}\right)$,

$$
\mathrm{d}:\left(\begin{array}{rc}
-{ }^{t} C & \stackrel{0}{\vdots} \\
& 0 \\
0 & 0 \\
0 & \vdots \\
0
\end{array}\right) .
$$

Les deux bases sont orthonormée, donc la matrice dans ces bases de la divergence $\delta: \Omega^{2}(M)^{G} \rightarrow \Omega^{1}(M)^{G}$ sera donc la transposée de la matrice ci-dessus.

Comme la différentielle restreinte à $\Omega^{0}(M)^{G}$ est nulle, le laplacien $\Delta=\delta \mathrm{d}+\mathrm{d} \delta$ se réduit sur $\Omega^{1}(M)^{G}$ à l'opérateur $\delta$ d. On en déduit la matrice du laplacien $\Delta^{i n v}$ restreint à $\Omega^{1}(M)^{G}$ est, dans la base $\left(V_{1}^{b}, \cdots, V_{n}^{b}, Y^{b}\right)$,

$$
\left(\begin{array}{cc}
-C & 0 \\
0 \cdots 0 & 0 \cdots 0
\end{array}\right)\left(\begin{array}{cc}
-{ }^{t} C & \stackrel{0}{\vdots} \\
& 0 \\
0 & \vdots \\
0 & 0
\end{array}\right)=\left(\begin{array}{cc}
C^{t} C & 0 \\
& \vdots \\
0 \cdots 0 & 0
\end{array}\right) .
$$

Remarque : On a fait ici le calcul pour un $Y$ fixé, c'est-à-dire pour un certain choix de connexion du fibré. Mais si on choisit $Y^{\prime}$ tel que $Y^{\prime}-Y \in \Gamma\left(T_{V} M\right)^{G}$ et une métrique telle que $\left(V_{1}, \cdots, V_{n}, Y^{\prime}\right)$ soit orthonormée, le résultat sera le même car on aura toujours $\left[Y^{\prime}, V_{i}\right]=\left[Y, V_{i}\right]=\sum_{j=1}^{n} b_{j i} V_{j}$. 


\subsection{Courbure}

Nous allons démontrer dans cette partie un lemme qui nous servira à faire le lien entre le contrôle de la courbure et l'existence de petites valeurs propres.

Lemme 2.6. Soir a la borne supérieure de la valeur absolue de la courbure sectionnelle de $(M, g)$. Il existe des constantes $\tau(n)>0$ et $\kappa(B)$ telle que

$$
\tau^{-1} a<\operatorname{Tr}\left(C^{t} C\right)<\tau a+\kappa .
$$

Démonstration : Rappelons tout d'abord l'expression suivante (dont le lecteur pourra trouver la démonstration dans [CE75]) de la courbure sectionnelle $K(U, V)$, où $U$ et $V$ sont deux champs invariants à gauche d'un groupe de Lie quelconque :

$$
\begin{aligned}
K(U, V)= & \frac{1}{4}\left\|\operatorname{ad}_{U}^{*} V+\operatorname{ad}_{V}^{*} U\right\|^{2}-\left\langle\operatorname{ad}_{U}^{*} U, \operatorname{ad}_{V}^{*} V\right\rangle \\
& -\frac{3}{4}\|[U, V]\|^{2}-\frac{1}{2}\langle[[U, V], V], U\rangle-\frac{1}{2}\langle[[V, U], U], V\rangle .
\end{aligned}
$$

Nous allons appliquer cette relation aux champs de la base $\left(V_{i}, Y\right)$. Pour cela, remarquons d'abord que les matrices de $\operatorname{ad}_{Y}$ et $\operatorname{ad}_{V_{i}}$ sont, dans cette base

$$
\operatorname{ad}_{Y}:\left(\begin{array}{cc}
C & 0 \\
\vdots \\
0 \cdots 0 & 0
\end{array}\right) \text { et } \operatorname{ad}_{V_{i}}:\left(\begin{array}{cc} 
& -c_{1 i} \\
0 & \vdots \\
0 \cdots 0 & -\dot{c}_{n i}
\end{array}\right)
$$

On en déduit $\operatorname{ad}_{V_{i}}^{*} Y=0, \operatorname{ad}_{Y}^{*} Y=0, \operatorname{ad}_{Y}^{*} V_{i}=\sum_{j} c_{i j} V_{j}$ et $\operatorname{ad}_{V_{i}}^{*} V_{j}=-c_{j i} Y$, et donc que

$$
\begin{aligned}
K\left(Y, V_{i}\right) & =\frac{1}{4}\left\|\operatorname{ad}_{Y}^{*} V_{i}\right\|^{2}-\frac{3}{4}\left\|\left[Y, V_{i}\right]\right\|^{2}-\frac{1}{2}\left\langle\left[\left[V_{i}, Y\right], Y\right], V_{i}\right\rangle \\
& =\frac{1}{4} \sum_{j}\left(c_{i j}^{2}-3 c_{j i}^{2}-2 c_{i j} c_{j i}\right)=-\sum_{j} c_{j i}^{2}+\frac{1}{4} \sum_{j}\left(c_{i j}-c_{j i}\right)^{2}
\end{aligned}
$$

et

$$
\begin{aligned}
K\left(V_{i}, V_{j}\right) & =\frac{1}{4}\left\|\operatorname{ad}_{V_{i}}^{*} V_{j}+\operatorname{ad}_{V_{j}}^{*} V_{i}\right\|^{2}-\left\langle\operatorname{ad}_{V_{i}}^{*} V_{i}, \operatorname{ad}_{V_{J}}^{*} V_{j}\right\rangle \\
& =\frac{1}{4}\left(c_{i j}+c_{j i}\right)^{2}-c_{i i} c_{j j} .
\end{aligned}
$$

D'autre part, comme $C$ est la matrice de $f$, le terme de degré $n-2$ du polynôme caractéristique est indépendant de la métrique choisie. Le calcul montre que son coefficient est $\kappa=\sum_{i j}\left(c_{i i} c_{j j}-c_{i j} c_{j i}\right)$. On peut en déduire que

$$
\sum_{i, j=1}^{n} K\left(V_{i}, V_{j}\right)+\kappa=\sum_{i, j=1}^{n}\left(\frac{1}{4}\left(c_{i j}+c_{j i}\right)^{2}-c_{i j} c_{j i}\right)=\frac{1}{4} \sum_{i, j=1}^{n}\left(c_{i j}-c_{j i}\right)^{2},
$$

et donc que

$$
\sum_{i, j=1}^{n} c_{j i}^{2}=\sum_{i, j=1}^{n} K\left(V_{i}, V_{j}\right)-\sum_{i=1}^{n} K\left(Y, V_{i}\right)+\kappa \leq\left(n^{2}+n\right) a+\kappa,
$$

ce qui montre l'une des deux inégalité du lemme. La seconde découle du fait que la courbure sectionnelle s'écrit comme un polynôme homogène de degré deux relativement aux $c_{i j}$. 


\subsection{Petites valeurs propres}

Nous allons maintenant démontrer les résultats concernant le spectre de $\Delta^{i n v}$.

Démonstration de 1.3.1 : Si $U$ est un vecteur colonne tel que $C^{t} C U=0$, alors ${ }^{t} U C^{t} C U=0$ et donc $\left\|{ }^{t} C U\right\|=0$. Par conséquent, $\operatorname{dim} \operatorname{Ker} C^{t} C=\operatorname{dim} \operatorname{Ker}{ }^{t} C=$ $\operatorname{dim} \operatorname{Ker} C=d^{\prime}$, et $\operatorname{dim} \operatorname{Ker} \Delta_{i n v}^{1}=d^{\prime}+1$.

Démonstration de 1.3.2 : Comme la trace de $\Delta_{i n v}^{1}$ est celle de $C^{t} C$, le résultat découle directement du lemme 2.6.

\section{Démonstration de 1.3.3 :}

Supposons que $d \neq n$. Soit $E_{0}$ le sous-espace caractéristique de $f$ associé à la valeur propre 0 . On notera $E_{0}^{\perp}$ son orthogonal pour la dualité dans l'espace des 1formes invariantes verticales. Comme $d \neq n$, l'espace $E_{0}^{\perp}$ est de dimension non nulle. On va montrer que le quotient de Rayleigh est uniformément minoré sur $E_{0}^{\perp}$, pour ensuite appliquer le principe du minimax.

Remarques : comme les formes et les métriques considérées sont invariantes, la norme ponctuelle d'une forme ne dépendra pas du point où on la calcule, ce qui permet d'écrire que $R(\alpha)=\frac{\|d \alpha\|^{2}}{\|\alpha\|^{2}}=\frac{|\mathrm{d} \alpha|^{2}}{|\alpha|^{2}}$. D'autre part, il faut noter que la notion d'orthogonalité pour la dualité est indépendante de la métrique. En particulier, comme $E_{0}$ est défini indépendamment de la métrique, $E_{0}^{\perp}$ le sera aussi.

Soit $V^{\mathrm{b}} \in E_{0}^{\perp}$ et $\left(V_{i}\right)$ une base de $\Gamma\left(T_{V} M\right)^{G}$ telle que $\left(V_{1}, \cdots, V_{d}\right)$ soit une base orthonormée de $E_{0}$ (si $d=0$ et donc $E_{0}=0$, on choisit alors $\left(V_{i}\right)$ orthonormée quelconque, la suite de la démonstration restant valide). L'espace $E_{0}$ est stable par $f$, donc $E_{0}^{\perp}$ est stable par ${ }^{t} f$, et la matrice de $\left({ }^{t} f\right)_{\mid E_{0}^{\perp}}$ dans la base $\left(V_{d+1}^{b}, \cdots, V_{n}^{b}\right)$ est ${ }^{t} D$, où $D$ est une sous-matrice de $C$. Comme la relation $(2.5)$ peut s'écrire $\mathrm{d} V^{b}=$ $-Y^{b} \wedge\left({ }^{t} f\right)\left(V^{b}\right)$, on a $\left|\mathrm{d} V^{b}\right|^{2}=\left|\left({ }^{t} f\right)\left(V^{b}\right)\right|^{2} \geq \lambda\left|V^{b}\right|^{2}$, où $\lambda$ est la plus petite valeur propre de $D^{t} D$. D'une part, le déterminant de cette matrice vérifie $\operatorname{Det} D^{t} D=$ $\left(\operatorname{Det}^{t} D\right)^{2}=\left(\operatorname{Det}\left({ }^{t} f\right)_{\mid E_{0}^{\perp}}\right)^{2}$, et donc Det $D^{t} D$ est indépendant du choix de la base $\left(V_{i}\right)$. D'autre part, $\operatorname{Det}^{t}\left(f_{\mid E_{0}^{\perp}}\right)$ est non nul. En effet, si ${ }^{t} f_{\mid E_{0}^{\perp}}(\alpha)=0$, alors $\alpha \circ f=0$, donc $\alpha$ est orthogonal à l'image de $f$, qui contient les sous-espaces caractéristiques de $f$ autres que $E_{0}$, et par conséquent $\alpha$ est nul. On en déduit que $\lambda$ est uniformément minorée : s'il existe une suite de métriques telle que $\lambda \rightarrow 0$, alors la plus grande valeur propre de $D^{t} D$ tend vers l'infini (car Det $D^{t} D$ est constant), ce qui est impossible puisque la courbure est bornée et que $\operatorname{Tr} C^{t} C \geq \operatorname{Tr} D^{t} D$ (car $D$ est une sous-matrice de $C$ ), et donc que la somme des valeurs propres de $D^{t} D$ est bornée.

On a montré que le quotient de Rayleigh de $\alpha \in E_{0}^{\perp}$ est minoré par une constante $c(f, a)$ indépendante de la métrique et du choix de $\alpha$. Comme $\operatorname{dim} E_{0}^{\perp}=n-d$, le principe du minimax nous dit donc que les $n+1-d$ plus grandes valeurs propres de $\Delta_{i n v}^{1}$ sont minorée par $c$. Comme $\operatorname{dim} \operatorname{Ker} \Delta_{i n v}^{1}=d^{\prime}+1$ et que $\operatorname{dim} \Omega^{1}(M)^{G}=n+1$, on en déduit que $\lambda_{d-d^{\prime}+1,1}^{i n v}>c$.

Si $d=n$, alors il existe $P \in \mathrm{GL}_{n}(\mathbb{R})$ tel que $P^{-1} B P$ soit triangulaire supérieure avec des 0 sur la diagonale, et comme $P^{-1} A P=P^{-1} \exp (B) P=\exp \left(P^{-1} B P\right)$, la matrice $P^{-1} A P$ sera triangulaire supérieure avec des 1 sur la diagonale. On en déduit, en posant $P^{\prime}=\left(\begin{array}{cc}P & 0 \\ 0 & I\end{array}\right) \in \mathrm{GL}_{n+2}(\mathbb{R})$, que le groupe $P^{\prime-1} G P^{\prime}$, où $G$ est le groupe construit au paragraphe 2.1i, est constitué de matrices triangulaires supérieures avec des 1 sur la diagonale. C'est donc un groupe nilpotent. 
L'existence d'un effondrement tel que toutes les valeurs propres de $\Delta_{i n v}^{1}$ tendent vers zéro découlera du 1.3 .4

Démonstration de 1.3.4 : On vient de démontrer que si $d$ (et donc $d^{\prime}$ ) est nul, il n'y a pas de petites valeurs propres.

Supposons que $d>0$. Pour simplifier, nous allons montrer le résultat dans le cas où la partie nilpotente de la réduite de Jordan de $B$ ne comporte qu'un seul bloc de Jordan, la construction de $g_{\varepsilon}^{k}$ étant semblable dans le cas général.

On construit une base $\left(V_{1}, \cdots, V_{n}\right)$ de $\Gamma\left(T_{V} M\right)^{G}$ en choisissant une base de Jordan $\left(V_{1}, \cdots, V_{d}\right)$ de $E_{0}$ (en particulier, $\left(V_{1}, \cdots, V_{d^{\prime}}\right)$ sera une base de Ker $f$ ), et on la complète de manière quelconque en une base de $\left(V_{1}, \cdots, V_{d^{\prime}}\right)$. On notera $C$ la matrice de $f$ dans cette base. La matrice $C$ n'est pas de Jordan, mais sa restriction à $E_{0}$, c'est-à-dire le bloc carré supérieur droit de taille $d$, l'est. Elle est de la forme

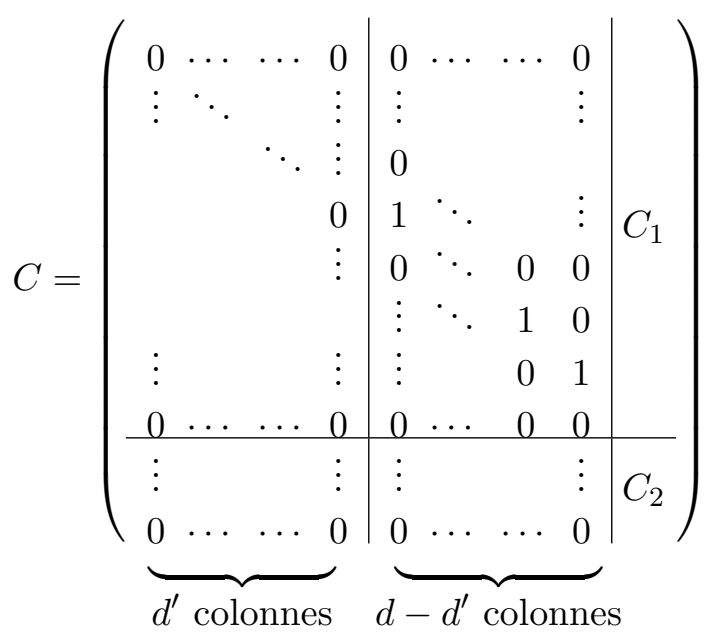

où $C_{2}$ est un bloc carré de taille $n-d$ et de déterminant non nul.

Soit $k \leq d-d^{\prime}$. On pose $V_{i}^{\varepsilon}=\nu_{i}(\varepsilon) V_{i}$, avec $\nu_{i}(\varepsilon)=\varepsilon^{-1}$ pour $i \geq d^{\prime}+k$, et $\nu_{i}(\varepsilon)=\varepsilon^{-\left(1+d^{\prime}+k-i\right)} V_{i}$ pour $i<d^{\prime}+k$. La matrice $C_{\varepsilon}$ de $f$ dans cette base vérifiera

$$
c_{i j}^{\varepsilon}=\frac{\nu_{j}(\varepsilon)}{\nu_{i}(\varepsilon)} c_{i j},
$$

donc $c_{i j}^{\varepsilon}=c_{i j}$ pour $i \geq d^{\prime}+k$ (en tenant compte du fait que $c_{i j}=0$ pour $i \geq d$ et $j \leq d)$, et $c_{i j}^{\varepsilon} \rightarrow 0$ quand $\varepsilon \rightarrow 0$, pour $i<d^{\prime}+k$. La matrice $C_{\varepsilon}$ tend donc vers une matrice $C_{0}$ de la forme

$$
C_{0}=\left(\begin{array}{cccc|cccc|c}
0 & \cdots & \cdots & 0 & 0 & \cdots & \cdots & 0 & \\
\vdots & \ddots & & \vdots & \vdots & & & \vdots & 0 \\
& & \ddots & \vdots & 0 & & & & \\
& & & 0 & 1 & \ddots & & \vdots & \\
& & & \vdots & 0 & \ddots & 0 & 0 & \\
& & & & \vdots & \ddots & 1 & 0 & C_{1}^{\prime} \\
\vdots & & & \vdots & \vdots & & 0 & 1 & \\
0 & \cdots & \cdots & 0 & 0 & \cdots & \cdots & 0 & \\
\hline \vdots & & & \vdots & \vdots & & & \vdots & C_{2}^{\prime} \\
0 & \cdots & \cdots & 0 & 0 & \cdots & \cdots & 0 &
\end{array}\right)
$$




$$
d^{\prime}+k \text { colonnes } \underbrace{}_{d-d^{\prime}-k \text { colonnes }}
$$

Comme les $\lambda_{i, 1}^{i n v}$ sont ces fonctions continues de $C$, il suffit de calculer la dimension du noyau de $C_{0}{ }^{t} C_{0}$, qui est égale à celle de $\operatorname{Ker} C_{0}$. D'une part, les $d^{\prime}+k$ premières colonnes de $C_{0}$ sont nulles, donc $\operatorname{dim} \operatorname{Ker} C_{0} \geq d^{\prime}+k$, et d'autre part, comme $\operatorname{det} C_{2} \neq$ 0 , la famille formée par les lignes $d^{\prime}+k$ à $d-1$ et les $n-d$ dernières lignes de $C_{0}$ est libre, donc $\operatorname{dim} \operatorname{Ker} C_{0} \leq n-(n-d)-\left((d-1)-\left(d^{\prime}+k-1\right)\right)=d^{\prime}+k$. De même, $\operatorname{dim} \operatorname{Ker} C=d^{\prime}$, donc on a bien exactement $k$ petites valeurs propres.

Si la partie nilpotente de la réduite de Jordan de $B$ contient plusieurs blocs de Jordan, on obtient le résultat en procédant de la même manière pour annuler le nombre souhaité de lignes dans $C$.

Remarques : la famille de matrice $C_{\varepsilon}$ est uniformement bornée par rapport à $\varepsilon$, et le lemme 2.6 donne la majoration $\left|K\left(M, g_{\varepsilon}^{k}\right)\right| \leq \tau \operatorname{Tr}\left(C_{\varepsilon}{ }^{t} C_{\varepsilon}\right)$, pour tout $\varepsilon$. La courbure sectionnelle du fibré est donc bien uniformément bornée au cours de l'effondrement. D'autre part, on voit que si on effondre le fibré par homothétie de la fibre, par exemple en posant $\nu_{i}(\varepsilon)=\varepsilon^{-1}$ pour tout $i$, la matrice $C_{\varepsilon}$ est indépendante de $\varepsilon$, et donc il n'y a pas de petites valeurs propres.

Démonstration du corollaire 1.6 : $\mathrm{Si} d=d^{\prime}$ et $d \neq n$, alors $\lambda_{1,1}^{i n v}$ est uniformément minoré d'après 1.3.3. Si $d=d^{\prime}$ et $d=n$, alors $B=0$ et toutes les valeurs propres de $\Delta_{i n v}^{1}$ sont nulles.

Si $d \neq d^{\prime}$, alors 1.3.4 garantit l'existence d'une petite valeur propre.

Démonstration du théorème $\mathbf{1 . 1 0}$ : Comme $B$ est semi-simple, son orbite par conjugaison est fermée ([CMG93], p. 28). Comme la courbure est bornée, la norme de $C$ reste bornée quand la base $\left(V_{1}^{b}, \cdots, V_{n}^{b}, Y^{b}\right)$ - et donc la métrique varie. La matrice $C$ est par construction dans l'orbite par conjugaison de $B$, donc elle prend finalement ses valeurs au cours de l'effondrement dans une partie compacte $K$ de cet orbite.

La base orthonormée $\left(V_{1}^{b}, \cdots, V_{n}^{b}, Y^{b}\right)$ de $\Omega^{1}(M)^{G}$ engendre, par produit extérieur, une base orthonormée de $\Omega^{*}(M)^{G}$. Les coefficients de la matrice de la différentielle extérieure $d$ dans cette base, et donc ceux de la matrice de $\Delta=\mathrm{d} \delta+\delta \mathrm{d}$, sont des fonctions continues de $C \subset K$. Par consq́uent, quand la métrique varie, la matrice de $\Delta$ prend ses valeurs dans un compact image de $K$. S'il existe une famille de métrique telle que $\lambda_{1, p}^{i n v}$ tende vers zéro pour un $p \in[1, n]$, alors la matrice de $\Delta$ tend vers une matrice de rang strictement inférieur, ce qui est impossible puisque, par compacité, la matrice limite sera dans l'image de $K$, donc de même rang que $\Delta$.

Par conséquent, l'opérateur $\Delta$ restreint à $\Omega^{*}(M)^{G}$ n'admet pas de petites valeurs propres.

Démonstration du corollaire 1.11 : Montrons d'abord que s'il existe $p$ tel que $\lambda_{1, p}^{i n v} \rightarrow 0$, alors $d \neq d^{\prime}$.

Si $p=1$, cela découle du corollaire 1.6. Si $p=n$, on est ramené à la situation $p=1$ par dualité de Hodge.

Reste le cas $p=2$ et $n=3$. On a déjà calculé les matrices de $\delta: \Omega^{2}(M)^{G} \rightarrow$ $\Omega^{1}(M)^{G}$ et d $: \Omega^{1}(M)^{G} \rightarrow \Omega^{2}(M)^{G}$. On en déduit que la matrice de $\delta$ d, en restriction à $\Omega^{2}(M)^{G}$ est de la forme, dans les bases introduites au paragraphe 2.2,

$$
\mathrm{d} \delta:\left(\begin{array}{cc}
{ }^{t} C C & 0 \\
0 & 0
\end{array}\right) \text {. }
$$


Comme la variété est de dimension 4, l'opérateur de Hodge $*$ est une isométrie de $\Omega^{2}(M)^{G}$. En restriction à $\Omega^{2}(M)^{G}$, on aura $\delta \mathrm{d}=* \mathrm{~d} \delta *$, et donc $\delta \mathrm{d}$ et $\mathrm{d} \delta$ ont même spectre. D'autre part, d'après la théorie de Hodge, le spectre du laplacien est la réunion des spectres de $\delta \mathrm{d}$ et $\mathrm{d} \delta$, on déduit de ce qui précède qu'une petite valeur propre non nulle de $\Delta_{i n v}^{2}$ sera une petite valeur propre non nulle de $\mathrm{d} \delta_{\mid \Omega^{2}(M)^{G}}$, et donc une petite valeur propre de ${ }^{t} C C$. Le raisonnement appliqué à $C^{t} C$ dans la démonstration de 1.3.4 reste valable pour ${ }^{t} C C$. On peut donc conclure que si $\lambda_{1,2}^{i n v}$ tend vers zéro, alors $d \neq d^{\prime}$.

Supposons que $d \neq d^{\prime}$. Alors le noyau de $B$ est non trivial, par conséquent $d>$ $d^{\prime}>0$ et la multiplicité de la valeur propre 0 de $B$ est au moins égale à deux. Si $n=3$ la troisième valeur propre est égale à la trace de $B$ qui est nulle puisqu'elle est réelle et que $\exp (\operatorname{Tr} B)=\operatorname{det}(\exp B)=\operatorname{det} A=1$. Donc $d=n$, et $G$ est nilpotent, d'après 1.3.3.

\section{Fibrés principaux en tore sur $T^{2}$}

\subsection{Démonstration du théorème $\mathbf{1 . 1 3}$}

Démonstration de 1.13.1 : Soit $M$ un fibré principal de base $T^{2}$ et de fibre $F$. La base du fibré peut s'écrire

$$
[0,1] \times[0,1] / \sim,
$$

où $\sim$ est la relation d'équivalence engendrée par $(x, 0) \sim(x, 1)$ et $(0, y) \sim(1, y)$. Le fibré $M$ peut alors se définir par la donnée, pour tout point $p$ du bord $\partial K$ de $K=[0,1] \times[0,1]$ d'un difféomorphisme $\varphi_{p}$ de la fibre, et en posant

$$
M=K \times F_{/(p, x) \sim\left(q, \varphi_{q}^{-1} \circ \varphi_{p}(x)\right), \forall x \in F, \forall p, q \in \partial K, p \sim q} .
$$

L'hypothèse de principalité se traduit ici par le fait que pour tout $p, q \in \partial K$ tels que $p \sim q$ et pour tout $g, x \in F$, on a

$$
(p, g \cdot x) \sim\left(q, g \cdot \varphi_{q}^{-1} \circ \varphi_{p}(x)\right),
$$

ce qui impose aux $\varphi_{q}^{-1} \circ \varphi_{p}$ d'être des translations à droite sur la fibre. On peut donc, sans perte de généralité se restreindre, pour le choix des $\varphi_{p}$, au groupe des translations de la fibre, qui est isomorphe à $F$. Le fibré est donc déterminé par la donnée d'une application de $\partial K$ dans $F$. Comme sa topologie ne dépend pas de la classe d'homotopie de cette application, l'ensemble des fibrés principaux de fibre $F$ sur le tore $T^{2}$ est parametré par le groupe fondamental de $F$. Il s'agit en fait d'un exemple de classe d'obstruction ([St51]) qui est, dans le cas général d'un $F$-fibré principal sur une variété compacte $N$ un élément de $H_{2}\left(N, \pi_{1}(F)\right)$ et qui mesure l'obstruction du fibré à être trivial.

Considérons maintenant un fibré principal $M$ de fibre $T^{n}$, et $\left(a_{1}, \cdots, a_{n}\right) \in$ $\pi_{1}\left(T^{n}\right)=\mathbb{Z}^{n}$ sa classe d'obstruction. Nous allons munir $\mathbb{R}^{n+2}$ d'une structure de groupe telle que la topologie du quotient à gauche par $\mathbb{Z}^{n+2}$ soit celle du fibré. Pour ce faire, nous choisirons le représentant $\gamma: \partial K \rightarrow T^{n}$ de la classe d'obstruction de 
la manière suivante :

$$
\begin{gathered}
\gamma_{\mid\{0\} \times[0,1]}=\gamma_{\mid[0,1] \times\{0\}}=\gamma_{\mid[0,1] \times\{1\}}=0, \\
\gamma(1, t)=\left(t a_{1}, \cdots, t a_{n}\right), \forall t \in[0,1],
\end{gathered}
$$

de sorte qu'un élément $\left(x_{1}, \cdots, x_{n}\right) \in T^{n}$ de la fibre au dessus de $(0, t) \in K$ sera identifié à l'élément $\left(x_{1}+t a_{1}, \cdots, x_{n}+t a_{n}\right)$ au dessus de $(1, t)$. Si on note $\left(x_{1}, \cdots, x_{n}, y_{1}, y_{2}\right)$ les éléments de $\mathbb{R}^{n+2}$, on veut donc définir sur cet ensemble un produit tel que

$$
\left(k_{1}, \cdots, k_{n}, 0,0\right) \cdot\left(x_{1}, \cdots, x_{n}, y_{1}, y_{2}\right)=\left(x_{1}+k_{1}, \cdots, x_{n}+k_{n}, y_{1}, y_{2}\right)
$$

de sorte que d'une part les sous-espaces de $\mathbb{R}^{n+2}$ d'équation $\left(y_{1}, y_{2}\right)=c^{t e}$ passent au quotient comme des tores, et tel que

$$
\begin{aligned}
& \left(0, \cdots, 0, l_{1}, l_{2}\right) \cdot\left(x_{1}, \cdots, x_{n}, y_{1}, y_{2}\right)= \\
& \left(x_{1}+y_{2} a_{1} l_{1}, \cdots, x_{n}+y_{2} a_{n} l_{1}, y_{1}+l_{1}, y_{2}+l_{2}\right)
\end{aligned}
$$

de sorte que la structure de fibré en tore sera bien celle définie par (3.1).

On peut effectivement construire une telle structure de groupe en plongeant $\mathbb{R}^{n+2}$ dans $M_{n+3}(\mathbb{R})$ par l'application suivante :

$$
\left(x_{1}, \cdots, x_{n}, y_{1}, y_{2}\right) \longmapsto\left(\begin{array}{c|ccc}
\multirow{2}{*}{I_{n}} & 0 & a_{1} y_{1} & x_{1} \\
& \vdots & \vdots & \vdots \\
& 0 & a_{n} y_{1} & x_{n} \\
\hline \multirow{2}{*}{0} & 1 & 0 & y_{1} \\
0 & 0 & 1 & y_{2} \\
0 & 0 & 1
\end{array}\right)
$$

Notons $G$ l'image de cette application. C'est un sous-groupe de $M_{n+3}(\mathbb{R})$, et le quotient $\Gamma \backslash G$ où $\Gamma$ est le réseau des entiers de $G$ est difféomorphe à la variété $M$, qui est donc une nilvariété.

Supposons maitenant que $n \geq 2$. On pose $d=\operatorname{pgcd}\left(a_{1}, \cdots, a_{n}\right)$ et $a_{i}^{\prime}=a_{i} / d$. Soit $P=\left(p_{i j}\right) \in M(n, \mathbb{Z})$ une matrice telle que $p_{i 1}=a_{i}^{\prime}$ et que ses vecteurs colonnes forment une base du réseau $\mathbb{Z}^{n}$. On a alors

$$
P^{-1}\left(\begin{array}{c}
a_{1} \\
\vdots \\
a_{n}
\end{array}\right)=\left(\begin{array}{c}
d \\
0 \\
\vdots \\
0
\end{array}\right)
$$

et

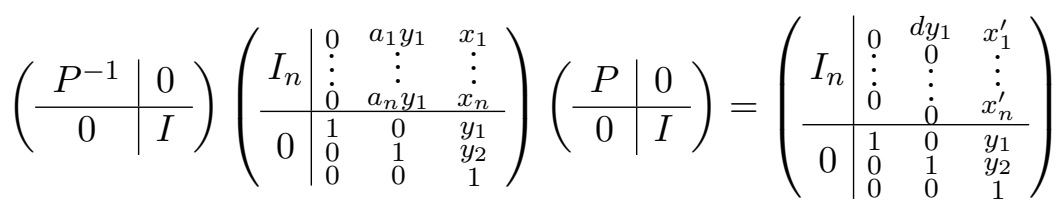

avec $\left(\begin{array}{c}x_{1}^{\prime} \\ \vdots \\ x_{n}^{\prime}\end{array}\right)=P^{-1}\left(\begin{array}{c}x_{1} \\ \vdots \\ x_{n}\end{array}\right)$. On peut voir que le groupe $P^{\prime-1} G P^{\prime}$, avec $P^{\prime}=\left(\begin{array}{cc}P & 0 \\ 0 & I\end{array}\right)$, est isomorphe à $\mathbb{R}^{n-1} \times G^{\prime}$, où $G^{\prime}$ est le groupe

$$
\left\{\left(\begin{array}{cccc}
1 & 0 & d y_{1} & x \\
0 & 1 & 0 & y_{1} \\
0 & 0 & 1 & y_{2} \\
0 & 0 & 0 & 1
\end{array}\right), x, y_{1}, y_{2} \in \mathbb{R}\right\}
$$


D'autre part, comme $\operatorname{det} P^{\prime}=1$ et que $P^{\prime}$ est à coefficients entiers, le réseau des matrices à coefficients entiers de $P^{\prime-1} G P^{\prime}$ est exactement $P^{\prime-1} \Gamma P^{\prime}$, où $\Gamma$ est le réseau des entiers de $G$. La variété $M=\Gamma \backslash G$, qui est difféomorphe à $P^{\prime-1} \Gamma P^{\prime} \backslash P^{\prime-1} G P^{\prime}$ peut donc s'écrire

$$
M \simeq\left(\mathbb{Z}^{n-1} \times \Gamma^{\prime}\right) \backslash\left(\mathbb{R}^{n-1} \times G^{\prime}\right) \simeq T^{n-1} \times N,
$$

où $N=\Gamma^{\prime} \backslash G^{\prime}$, en notant $\Gamma^{\prime}=$ le réseau des entiers de $G^{\prime}$.

Ce calcul montre qu'on peut se ramener au cas où les $a_{i}, i \geq 2$ sont nuls. On supposera dans la suite que c'est le cas, et on posera $a_{1}=a$.

Démonstration de 1.13.2 : Soient $X_{i}, Y_{1}$ et $Y_{2}$ les champs de vecteur invariants à gauche engendrés en $I_{n+3}$ respectivement par

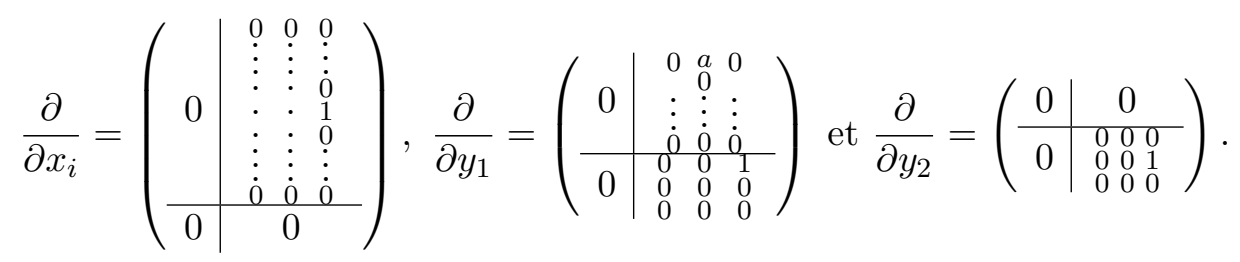

Ces champs vérifient $\left[X_{i}, X_{j}\right]=0,\left[X_{i}, Y_{j}\right]=0$ et $\left[Y_{1}, Y_{2}\right]=a X_{1}$. On notera $V$ le vecteur $a X_{1}$, dont on peut remarquer qu'il est non nul (si $a$ est nul, le fibré est trivial).

Soit $g$ une métrique homogène sur $M$, et $\left(V_{i}\right)$ une base de $\Gamma\left(T_{V} M\right)^{G}$ telle que $\left(V_{1}, \cdots, V_{n}, Y_{1}, Y_{2}\right)$ soit orthonormée en tout point et que $V_{1}$ soit colinéaire à $V$. Les crochets de Lie entre les vecteurs de cette base sont :

$$
\left[V_{i}, V_{j}\right]=0,\left[V_{i}, Y_{j}\right]=0 \text {, et }\left[Y_{1}, Y_{2}\right]=\eta V_{1}, \eta \in \mathbb{R}^{*} .
$$

On en déduit :

$$
\mathrm{d} V_{1}^{b}=-\eta Y_{1}^{\mathrm{b}} \wedge Y_{2}^{\mathrm{b}} \text { et } \mathrm{d} V_{i}^{\mathrm{b}}=\mathrm{d} Y_{j}^{\mathrm{b}}=0, i>1,
$$

où $\left(V_{1}^{b}, \cdots, V_{n}^{b}, Y_{1}^{b}, Y_{2}^{b}\right)$ est la base duale de $\left(V_{1}, \cdots, V_{n}, Y_{1}, Y_{2}\right)$. Les formes de cette base de $\Omega^{1}(M)^{G}$ engendrent, par produit exterieur, une base de $\Omega^{*}(M)^{G}$ composée de formes propres de $\Delta_{i n v}$. En effet, il découle de (3.5) qu'elles sont toutes fermées sauf celles de la forme $V_{1} \wedge V_{i_{1}} \wedge \cdots \wedge V_{i_{k}}\left(i_{j} \neq 1\right)$, dont la différentielle vaut:

$$
\mathrm{d}\left(V_{1} \wedge V_{i_{1}} \wedge \cdots \wedge V_{i_{k}}\right)=-\eta Y_{1} \wedge Y_{2} \wedge V_{i_{1}} \wedge \cdots \wedge V_{i_{k}}
$$

et, puisque $\delta=(-1)^{n(p+1)+1} * \mathrm{~d} *$, elles sont toutes cofermées sauf celles de la forme $Y_{1} \wedge Y_{2} \wedge V_{1} \wedge V_{i_{1}} \wedge \cdots \wedge V_{i_{k}}$ dont la codifférentielle vaut :

$$
\delta\left(Y_{1} \wedge Y_{2} \wedge V_{1} \wedge V_{i_{1}} \wedge \cdots \wedge V_{i_{k}}\right)=-\eta V_{1} \wedge V_{i_{1}} \wedge \cdots \wedge V_{i_{k}}
$$

En restriction à $\Omega^{p}(M)^{G}$, les formes de la base sont donc harmoniques, sauf $C_{n-1}^{p-1}$ formes cofermées et $C_{n-1}^{p-2}$ formes fermées qui sont des formes propres de valeur propre égale à $\eta^{2}$. L'opérateur $\Delta_{i n v}^{p}$ admet donc une unique valeur propre non nulle, de multiplicité $C_{n-1}^{p-1}+C_{n-1}^{p-2}=C_{n}^{p-1}$ et égale à $\eta^{2}=|V|^{2}$.

Si on choisit une base orthonormée de la forme $\left(V_{1}, \cdots, V_{n}, Y_{1}^{\prime}, Y_{2}^{\prime}\right)$, avec $Y_{i}^{\prime}=$ $Y_{i}+\sum_{k=1}^{n} \xi_{k} V_{k}$, on aura toujours $\left[Y_{1}^{\prime}, Y_{2}^{\prime}\right]=\left[Y_{1}, Y_{2}\right]$. Le résultat ne dépend donc pas 
du choix de la connexion sur le fibré. Remarquons enfin que si on choisit une autre métrique sur la base (en se donnant deux champs horizontaux quelconques $Y_{1}^{\prime}$ et $Y_{2}^{\prime}$ et en les supposant orthogonaux) on obtiendra le même résultat en remplaçant $V$ par $V^{\prime}=\left[Y_{1}^{\prime}, Y_{2}^{\prime}\right]$, avec comme valeur propre $\eta^{2}=\left|V^{\prime}\right|^{2}=\operatorname{Vol}(B)^{-2}|V|^{2}$.

\section{Exemples et remarques}

\subsection{Petites valeurs propres pour les 2-formes d'un fibré sur le cercle}

Nous allons donner ici un exemple de fibré en tore sur le cercle pour lequel $d=d^{\prime}=0$ et $\Delta_{i n v}^{2}$ admet une petite valeur propre. Cet exemple montre que le corollaire 1.6 ne se généralise pas à $n$ et $p$ quelconque.

On définit le fibré considéré par la matrice

$$
A=\left(\begin{array}{c|c}
A^{\prime} & A^{\prime \prime} \\
\hline 0 & A^{\prime}
\end{array}\right)
$$

avec

$$
A^{\prime}=\left(\begin{array}{ll}
2 & 1 \\
1 & 1
\end{array}\right) \text { et } A^{\prime \prime}=\left(\begin{array}{ll}
0 & 1 \\
0 & 0
\end{array}\right) .
$$

Fait 1 : La matrice $A$ est semblable à une matrice de la forme

$$
\left(\begin{array}{cc|cc}
e^{\lambda} & 1 & 0 & 0 \\
0 & e^{\lambda} & 0 & 0 \\
\hline 0 & 0 & e^{-\lambda} & 1 \\
0 & 0 & 0 & e^{-\lambda}
\end{array}\right)
$$

où $\lambda$ est un réel non nul.

Démonstration : La matrice $A^{\prime}$ admet deux valeurs propres réelles positives, qui sont inverses l'une de l'autre car Det $A^{\prime}=1$. On notera $\lambda$ le réel positif tel que ces deux valeurs propres soient $e^{\lambda}$ et $e^{-\lambda}$. Elles sont aussi valeurs propres de $A$ avec la multiplicité deux. On peut vérifier que le polynôme caractéristique de $A$ est son polynôme minimal. Les sous-espaces propres de $A$ sont donc tous les deux de dimension 1, et par conséquent, les deux blocs de sa réduite de Jordan sont $\left(\begin{array}{cc}e^{\lambda} & 1 \\ 0 & e^{\lambda}\end{array}\right)$ et $\left(\begin{array}{cc}e^{-\lambda} & 1 \\ 0 & e^{-\lambda}\end{array}\right)$.

Fait 2 : Il existe une suite de métrique $g_{\varepsilon}$ sur $M=\Gamma \backslash G(B)$ et une suite de matrices $C_{\varepsilon}$ associées telles que

$$
C_{\varepsilon}=\left(\begin{array}{cc|cc}
\lambda & \epsilon & 0 & 0 \\
0 & \lambda & 0 & 0 \\
\hline 0 & 0 & -\lambda & \epsilon \\
0 & 0 & 0 & -\lambda
\end{array}\right) .
$$

Démonstration : Comme on a

$$
\exp \left(\begin{array}{cc}
\lambda & e^{-\lambda} \\
0 & \lambda
\end{array}\right)=\left(\begin{array}{cc}
e^{\lambda} & 1 \\
0 & e^{\lambda}
\end{array}\right) \text { et } \exp \left(\begin{array}{cc}
-\lambda & e^{\lambda} \\
0 & -\lambda
\end{array}\right)=\left(\begin{array}{cc}
e^{-\lambda} & 1 \\
0 & e^{-\lambda}
\end{array}\right),
$$


La matrice $A$ admet un logarithme semblable à

$$
C=\left(\begin{array}{cc|cc}
\lambda & e^{-\lambda} & 0 & 0 \\
0 & \lambda & 0 & 0 \\
\hline 0 & 0 & -\lambda & e^{\lambda} \\
0 & 0 & 0 & -\lambda
\end{array}\right)
$$

Soit $\left(V_{1}, V_{2}, V_{3}, V_{4}\right)$ la base dans laquelle la matrice de l'endomorphisme $f$ est égal à $C$. Si on pose $V_{i}^{\varepsilon}=\varepsilon^{\alpha} V_{i}$ pour $i=1,3, V_{2}^{\varepsilon}=\varepsilon^{\alpha+1} e^{\lambda} V_{2}$ et $V_{4}^{\varepsilon}=\varepsilon^{\alpha+1} e^{-\lambda} V_{4}$, la matrice de $f$ dans cette base sera

$$
C_{\varepsilon}=\left(\begin{array}{cc|cc}
\lambda & \epsilon & 0 & 0 \\
0 & \lambda & 0 & 0 \\
\hline 0 & 0 & -\lambda & \epsilon \\
0 & 0 & 0 & -\lambda
\end{array}\right) .
$$

Il suffit donc de définir $g_{\varepsilon}$ en posant que la base $\left(V_{1}^{\varepsilon}, V_{2}^{\varepsilon}, V_{3}^{\varepsilon}, V_{4}^{\varepsilon}, Y\right)$ est orthonormée. Le fait que la courbure reste bornée quand $\varepsilon \rightarrow 0$ découle du lemme 2.6

Fait $3: \lambda_{1,2}^{i n v}\left(M, g_{\varepsilon}\right)$ tend vers zéro quand $\varepsilon \rightarrow 0$.

Démonstration :

On va calculer la matrice de $\mathrm{d}: \Omega^{2}(M)^{G} \rightarrow \Omega^{3}(M)^{G}$ dans des bases de la forme $\left(V_{i}^{\mathrm{b}} \wedge V_{j}^{\mathrm{b}}, V_{i}^{\mathrm{b}} \wedge Y^{\mathrm{b}}\right)$ et $\left(V_{i}^{\mathrm{b}} \wedge V_{j}^{\mathrm{b}} \wedge Y^{\mathrm{b}}, V_{i}^{\mathrm{b}} \wedge V_{j}^{\mathrm{b}} \wedge V_{k}^{\mathrm{b}}\right)$.

En utilisant (2.5), on obtient que $\mathrm{d} V_{i}^{\mathrm{b}} \wedge Y^{\mathrm{b}}=0$ pour tout $i$, et que

$$
\begin{aligned}
\mathrm{d}\left(V_{1}^{b} \wedge V_{2}^{b}\right) & =\left(\lambda V_{1}^{b}+\varepsilon V_{2}^{b}\right) \wedge Y^{b} \wedge V_{2}^{b}-V_{1}^{b} \wedge \lambda V_{2}^{b} \wedge Y^{b} \\
& =-2 \lambda V_{1}^{b} \wedge V_{2}^{b} \wedge Y^{b}, \\
\mathrm{~d}\left(V_{1}^{b} \wedge V_{3}^{b}\right) & =\left(\lambda V_{1}^{b}+\varepsilon V_{2}^{b}\right) \wedge Y^{b} \wedge V_{3}^{b}-V_{1}^{b} \wedge\left(-\lambda V_{3}^{b}+\varepsilon V_{4}^{b}\right) \wedge Y^{b} \\
& =-\varepsilon V_{2}^{b} \wedge V_{3}^{b} \wedge Y^{b}-\varepsilon V_{1}^{b} \wedge V_{4}^{b} \wedge Y^{b}, \\
\mathrm{~d}\left(V_{1}^{b} \wedge V_{4}^{b}\right) & =\left(\lambda V_{1}^{b}+\varepsilon V_{2}^{b}\right) \wedge Y^{b} \wedge V_{4}^{b}-V_{1}^{b} \wedge\left(-\lambda V_{4}^{b}\right) \wedge Y^{b} \\
& =-\varepsilon V_{2}^{b} \wedge V_{4}^{b} \wedge Y^{b}, \\
\mathrm{~d}\left(V_{2}^{b} \wedge V_{3}^{b}\right) & =\lambda V_{2}^{b} \wedge Y^{b} \wedge V_{3}^{b}-V_{2}^{b} \wedge\left(-\lambda V_{3}^{b}+\varepsilon V_{4}^{b}\right) \wedge Y^{b} \\
& =-\varepsilon V_{2}^{b} \wedge V_{4}^{b} \wedge Y^{b}, \\
\mathrm{~d}\left(V_{2}^{b} \wedge V_{4}^{b}\right) & =\lambda V_{2}^{b} \wedge Y^{b} \wedge V_{4}^{b}-V_{2}^{b} \wedge\left(-\lambda V_{4}^{b}\right) \wedge Y^{b}=0, \\
\mathrm{~d}\left(V_{3}^{b} \wedge V_{4}^{b}\right) & =\left(-\lambda V_{3}^{b}+\varepsilon V_{4}^{b}\right) \wedge Y^{b} \wedge V_{4}^{b}-V_{3}^{b} \wedge\left(-\lambda V_{4}^{b}\right) \wedge Y^{b} \\
& =2 \lambda V_{3}^{b} \wedge V_{4}^{b} \wedge Y^{b} .
\end{aligned}
$$

La matrice de $d$ dans les bases

$$
\left(V_{1}^{\mathrm{b}} \wedge V_{2}^{\mathrm{b}}, V_{1}^{\mathrm{b}} \wedge V_{3}^{\mathrm{b}}, V_{1}^{\mathrm{b}} \wedge V_{4}^{\mathrm{b}}, V_{2}^{\mathrm{b}} \wedge V_{3}^{\mathrm{b}}, V_{2}^{\mathrm{b}} \wedge V_{4}^{\mathrm{b}}, V_{3}^{\mathrm{b}} \wedge V_{4}^{\mathrm{b}}, V_{i}^{\mathrm{b}} \wedge Y^{\mathrm{b}}\right)
$$

et

$$
\begin{aligned}
& \left(V_{1}^{\mathrm{b}} \wedge V_{2}^{\mathrm{b}} \wedge Y^{b}, V_{1}^{\mathrm{b}} \wedge V_{3}^{\mathrm{b}} \wedge Y^{\mathrm{b}}, V_{1}^{\mathrm{b}} \wedge V_{4}^{\mathrm{b}} \wedge Y^{\mathrm{b}}, V_{2}^{\mathrm{b}} \wedge V_{3}^{\mathrm{b}} \wedge Y^{b}\right. \\
& \left.V_{2}^{\mathrm{b}} \wedge V_{4}^{\mathrm{b}} \wedge Y^{\mathrm{b}}, V_{3}^{\mathrm{b}} \wedge V_{4}^{\mathrm{b}} \wedge Y^{\mathrm{b}}, V_{i}^{\mathrm{b}} \wedge V_{j}^{\mathrm{b}} \wedge V_{k}^{b}\right)
\end{aligned}
$$


est de la forme

$$
\left(\begin{array}{rrrrrr|r}
-2 \lambda & 0 & 0 & 0 & 0 & 0 & \\
0 & 0 & 0 & 0 & 0 & 0 & \\
0 & -\varepsilon & 0 & 0 & 0 & 0 & 0 \\
0 & -\varepsilon & 0 & 0 & 0 & 0 & \\
0 & 0 & -\varepsilon & -\varepsilon & 0 & 0 & \\
0 & 0 & 0 & 0 & 0 & 2 \lambda & \\
\hline & & 0 & & & & 0
\end{array}\right) .
$$

On voit que quand $\varepsilon$ tend vers zéro, cette matrice tend vers une matrice de rang strictement inférieur. On peut en déduire comme au paragraphe 2.4 que l'opérateur $\delta$ d admet une petite valeur propre, qui sera aussi petite valeur propre de $\Delta$.

\subsection{Effondrements des fibrés principaux sur le tore}

Dans cette partie, nous allons montrer que les fibrés construits dans la partie 3 peuvent admettre, si $n \geq 2$, un effondrement à diamètre et courbure bornés pour lequel $\lambda$ ne tend pas vers zéro.

Soit $M$ un tel fibré. Nous allons d'abord montrer le lemme suivant qui nous permettra de contrôler la courbure :

Lemme 4.8. Pour toute métrique homogène g sur $M$, la courbure sectionnelle de $M$ vérifie $|K(M, g)| \leq \frac{3}{4}|V|^{2}$.

\section{Démonstation :}

De (3.4), on peut rapidement déduire que $\operatorname{ad}_{V_{i}}=0$, et que $\operatorname{ad}_{Y_{i}}^{*} Y_{j}=0$ car pour tout vecteur $U,\left\langle\operatorname{ad}_{Y_{i}}^{*} Y_{j}, U\right\rangle=\left\langle Y_{j}, \operatorname{ad}_{Y_{i}} U\right\rangle=\left\langle Y_{j},\left[Y_{i}, U\right]\right\rangle=0$. De plus, comme $\left\langle\operatorname{ad}_{Y_{i}}^{*} V_{j}, U\right\rangle=\left\langle V_{j},\left[Y_{i}, U\right]\right\rangle$, on aura $\operatorname{ad}_{Y_{i}}^{*} V_{j}=0$ pour $j \neq 1, \operatorname{ad}_{Y_{1}}^{*} V_{1}=\mu Y_{2}$ et $\operatorname{ad}_{Y_{2}}^{*} V_{1}=-\mu Y_{1}$.

La formule (2.7) donne donc :

$$
\begin{gathered}
K\left(V_{i}, V_{j}\right)=0, K\left(Y_{1}, Y_{2}\right)=-\frac{3}{4}\left\|\left[Y_{1}, Y_{2}\right]\right\|^{2}, \\
K\left(V_{1}, Y_{i}\right)=\frac{\mu^{2}}{4}, \text { et } K\left(V_{i}, Y_{j}\right)=0 \text { pour } i \neq 1 .
\end{gathered}
$$

Comme d'une part $\mu^{2}=\|V\|^{2}$, et d'autre part $V=\left[Y_{1}, Y_{2}\right]$, la majoration du lemme en découle immédiatement.

Un effondrement à base fixe du fibré $M$ par des métriques homogènes est déterminé par une famille de bases $\left(V_{1}^{\varepsilon}, \cdots, V_{n}^{\varepsilon}\right)$ de $\Gamma\left(T_{V} M\right)^{G}$. Nous allons présenter ici des exemples d'effondrements associés à des familles de bases de la forme $\left(V_{1}^{\varepsilon}, \cdots, V_{n}^{\varepsilon}\right)=\left(\varepsilon^{-\alpha_{1}} V_{1}, \cdots, \varepsilon^{-\alpha_{n}} V_{n}\right)$, où $\left(V_{1}, \cdots, V_{n}\right)$ est une base fixée. Si $b_{i}$ et $b_{i}^{\varepsilon}$ sont les coefficients de $V$ dans les bases respectives $\left(V_{1}, \cdots, V_{n}\right)$ et $\left(V_{1}^{\varepsilon}, \cdots, V_{n}^{\varepsilon}\right)$, on aura

$$
b_{i}^{\varepsilon}=\varepsilon^{\alpha_{i}} b_{i} .
$$

Si $\alpha_{i}>0$, pour tout $i$, alors le diamètre de la fibre tend vers 0 , ainsi que les $b_{i}^{\varepsilon}$. On a donc un effondrement à courbure bornée du fibré sur la base $T^{2}$, et la valeur propre $\lambda=\|V\|^{2}=\sum_{i=1}^{n}\left(b_{i}^{\varepsilon}\right)^{2}$ tend vers zéro. 
On peut cependant construire des effondrements pour lesquels le comportement du spectre est différent. Supposons par exemple que $\alpha_{i}=0$, pour tout $i>1$ et $\alpha_{1}>0$, et que les composantes de $V_{1}$ dans la base $\left(X_{1}, \cdots, X_{n}\right)$ soient irrationnelles entre elles. Une droite de la fibre de direction $V_{1}$ sera donc dense dans la fibre, et par conséquent, il suffit que seul $\alpha_{1}$ soit non nul pour que la fibre s'effondre sur un point. On aura alors $b_{i}^{\varepsilon}=b_{i}$ pour $i>1$, et $b_{1}^{\varepsilon} \rightarrow 0$. La courbure reste donc bornée et $\lambda \rightarrow \sum_{i>1} b_{i}^{2} \neq 0$.

\subsection{Formes invariantes et spectre de $\Delta$}

Nous allons ici nous intéresser à un exemple de fibré en tore $T^{2}$ sur le cercle qui montre que la proposition 1.1 ne se généralise pas aux quotients de groupes résolubles.

Ce fibré sera en fait le fibré trivial (homéomorphe au tore $T^{3}$ ), mais nous ne le contruirons pas comme quotient de $\mathbb{R}^{3}$. En effet, nous poserons

$$
A=\left(\begin{array}{ll}
1 & 0 \\
0 & 1
\end{array}\right) \text { et } B=\left(\begin{array}{cc}
0 & 2 \pi \\
-2 \pi & 0
\end{array}\right) .
$$

Le groupe $G(B)$ correspondant est alors un groupe résoluble non abélien, mais le réseau $\Gamma$ (qui est aussi le groupe fondamental du fibré) est isomorphe à $\mathbb{Z}^{3}$. La topologie du fibré - déterminée par $A$ - est donc celle du tore $T^{3}$, dont le premier nombre de Betti est $b_{1}=3$. Cependant, d'après le théorème 1.3 , le noyau de $\Delta_{i n v}^{1}$ est de dimension 1 pour des métriques homogènes sur $M$, et donc $\operatorname{dim} \operatorname{Ker} \Delta_{i n v}^{1}<$ $\operatorname{dim} \operatorname{Ker} \Delta^{1}=b_{1}$. Cela signifie que les formes harmoniques du fibrés ne sont pas nécessairement invariantes pour la structure homogène induite par $G$. La solvariété $M$ ne vérifie donc pas la conclusion de la proposition 1.1.

\section{Références}

[CC90] B. Colbois et G. Courtois - « A note on the first non zero eigenvalue of the Laplacian acting on $p$-forms $\gg$, Manuscripta Math., 68 (2), p. 143-160, 1990.

[CC00] B. Colbois et G. Courtois - $\ll$ Petites valeurs propres des $p$-formes différentielles et classe d'Euler des $S^{1}$-fibrés $\gg$, Ann. scient. Éc. norm. sup., 33 (5), p. 611-645, 2000.

[CE75] J. CheEger et D. G. EBIn - Comparison Theorems in Riemannian Geometry, North-Holland Publishing Compagny, 1975.

[CMG93] D. Collingwood et W. MC Govern - Nilpotent orbits in semisimple Lie algebras, Van Nostrand Reinhold, 1993.

[CT97] S. Chanillo et F. TrÈves - « On the lowest eigenvalue of the Hodge Laplacian $\gg$, J. Differ. Geom., 45 (2), p. 273-287, 1997.

[Fo95] R. Forman - « Spectral Sequences and Adiabatic Limits $\gg$, Comm. Math. Phys., 168 (1), p. 57-116, 1995.

[Gr80] M. Gromov - « Paul Levy's isoperimetric inegality », prépublication IHÉS, 1980, publié dans dans [Gr99].

[Gr99] M. GROMov - Metric structures for Riemannian and non-Riemannian spaces, volume 152 de Progress in Mathematics, Birkhäuser, 1999.

[Hu66] D. Husemöller - Fibre Bundles, Springer Verlag, 1966. 
[Lo02] J. LotT $-\ll$ Collapsing and the differential form Laplacian $\gg$, Duke Math. J., 114 (2), p. 267-306, 2002, math.DG/9902111.

[LY80] P. LI et S.T. YAU - « Eigenvalues of a compact riemannian manifold $\gg$, Proceedings Symposium on Pure Math., pages 205-239, 1980.

[MM90] R. Mazzeo et R. Melrose - « The adiabatic limit, Hodge cohomology and Leray spectral sequence for a fibration $\gg$, J. Differ. Geom., 31 (1), p. 185-213, 1990.

[Pe89] X.-W. Peng - « Collapsing riemannian manifolds to the circle », Math. Z., 202 (2), p. 289-298, 1989.

[St51] N. Steenrod - The topology of fibre bundles, Princeton University Press, 1951.

[Tu97] W. Tuschmann - « Collapsing, solvmanifolds and infrahomogeneous spaces $\gg$, Differ. Geom. Appl., 7 (3), p. 251-264, 1997.

Pierre Jammes,

Université de Neuchâtel, Institut de mathématiques,

Rue Émile Argand 11, C.P. 2

CH-2007 Neuchâtel

Pierre. Jammes@unine.ch 